\title{
Computational fluid dynamics visualizes turbulent flow in the aortic root of a patient under continuous-flow left ventricular assist device support
}

Shohei Yoshida, MD, PhD, Koichi Toda, MD, PhD, Shigeru Miyagawa, MD, PhD, and

Yoshiki Sawa, MD, PhD

From the Department of Cardiovascular Surgery, Osaka University Graduate School of Medicine, Osaka, Japan. Disclosures: Authors have nothing to disclose with regard to commercial support.

Received for publication Nov 26, 2018; revisions received Jan 3, 2019; accepted for publication Jan 15, 2019; available ahead of print Feb 26, 2019.

Address for reprints: Yoshiki Sawa, MD, PhD, 2-2 Yamadaoka, Suita, Osaka, 565-0871 Japan (E-mail: sawa-p@ surg 1.med.osaka-u.ac.jp).

J Thorac Cardiovasc Surg 2020;159:e205-7

$0022-5223 / \$ 36.00$

Copyright (C 2019 by The American Association for Thoracic Surgery

https://doi.org/10.1016/j.jtcvs.2019.01.070

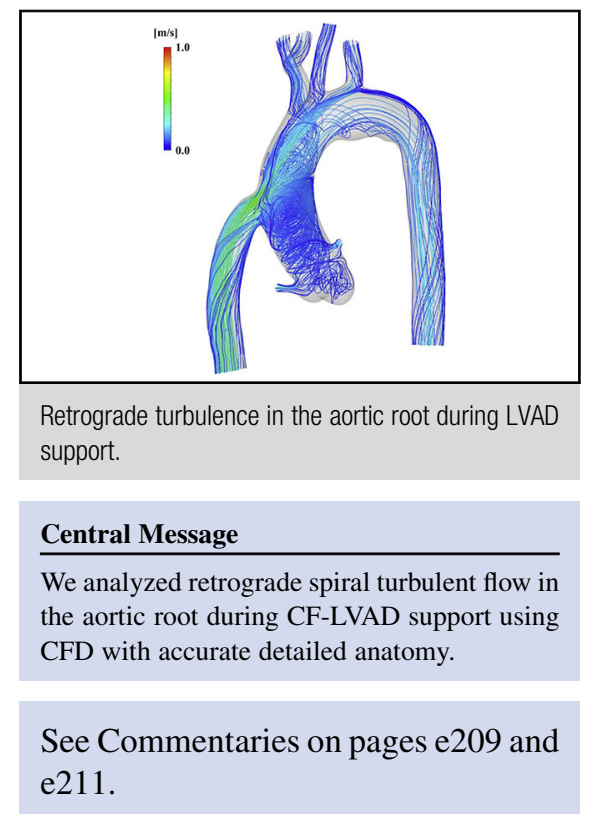

Aortic insufficiency (AI) developed during continuousflow left ventricular assist device (CF-LVAD) use occurs in $25 \%$ of patients within 1 year of CF-LVAD implantation. ${ }^{1,2}$ Nonphysiologic retrograde flow in the ascending aorta and shear stress in the aortic valve have been suggested to induce valveleaflet thinning, consequently, $\mathrm{AI}$ and its progression., Although blood flow analysis using vector flow mapping with 2-dimensional echocardiographic images or computational fluid dynamics (CFD) using the aortic mold without aortic root has been reported, blood flow and shear stress in the 3-dimensional aortic root with its complex anatomy are unknown. ${ }^{4,5}$ To evaluate the 3-dimensional blood flow, CFD analysis with an accurate aortic root anatomy, including the aortic valve and coronary arteries, was performed in a 40 -year-old woman in whom the aortic valve did not open after

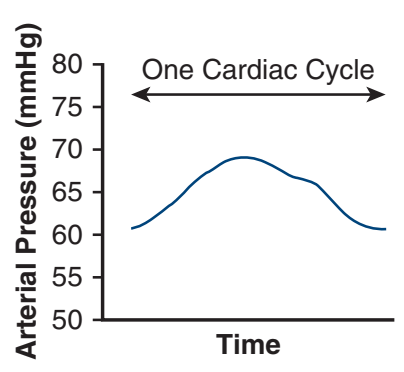

A

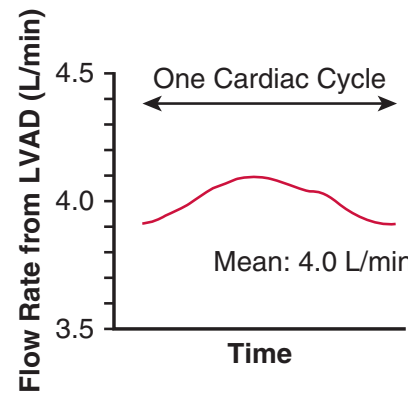

B
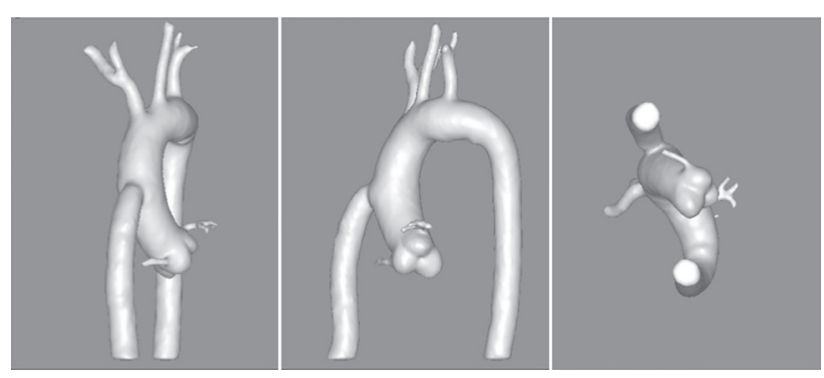

C

FIGURE 1. A, Graph representing the time-dependent change in the arterial pressure during 1 cardiac cycle in patients with ischemic cardiomyopathy under CF-LVAD support. B, Graph representing the time-dependent change in the flow rate from the LVAD during 1 cardiac cycle, which was calculated using the arterial pressure waveform and mean cardiac output $(4.0 \mathrm{~L} / \mathrm{min})$ in the Swan-Ganz catheter. C, Images showing a 3-dimensional patient-specific geometry of the aorta, the coronary and cervical arteries, and the outflow graft of the LVAD from the anterior (left), lateral (middle), and inferior (right) view. 

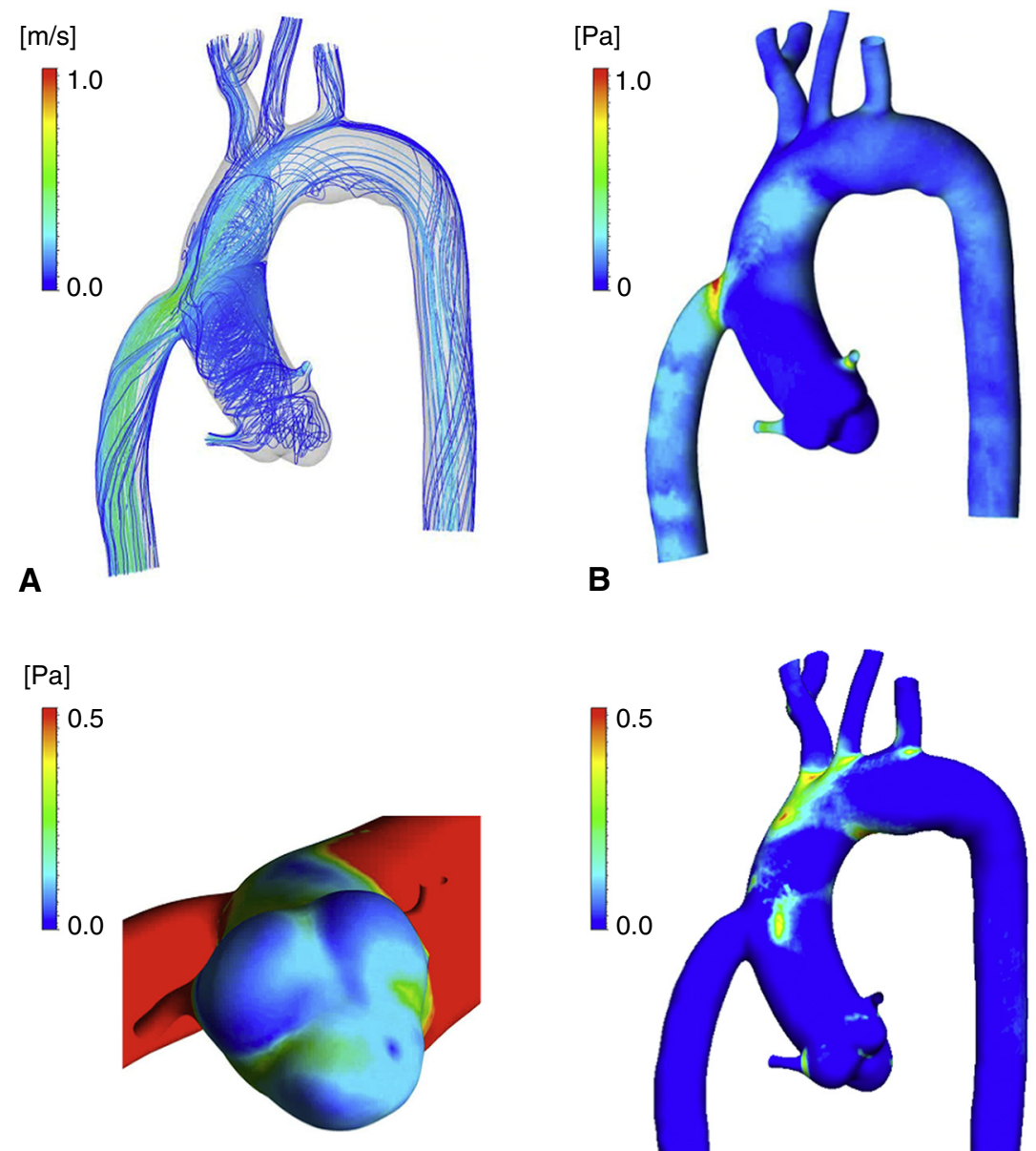

C

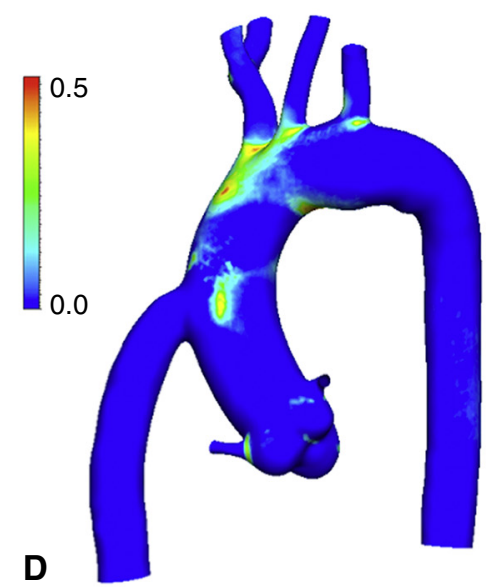

FIGURE 2. The results of CFD simulation in a patient supported with a CF-LVAD. A, Streamline shows retrograde spiral turbulence in the aortic root. B, Shear stress is low in the aortic root except in the coronary arteries. C, Shear stress is similar in each aortic valve cusp and relatively high in the commissures. $\mathrm{D}$, Oscillatory shear index in the aortic root is low.

CF-LVAD (HeartMate II, Abbott, Chicago, Ill) implantation for ischemic dilated cardiomyopathy. First, the accurate flow rate was calculated using the arterial pressure waveform and cardiac output in the Swan-Ganz catheter (Figure $1, A$ and $B$ ), and a 3-dimensional patient-specific geometry was created using AZE Virtual Place (AZE, Tokyo, Japan) with electrocardiogram-gated computed tomography images (Figure 1, C). Subsequently, CFD analysis, using ANSYS-FLUENT 18.0 (ANSYS, Inc, Canonsburg, $\mathrm{Pa}$ ), revealed retrograde spiral turbulence in the aortic root (Figure 2, A, Video 1), similar to a previous study that used echocardiography, ${ }^{4}$ and demonstrated a low shear stress in the aortic root excluding the coronary arteries (Figure 2, B, and Video 2). Shear stress was similar in each aortic valve cusp, whereas it was relatively high in the commissures (Figure 2, $C$, and Video 3). The oscillatory shear index in the aortic root was low (Figure 2, D).

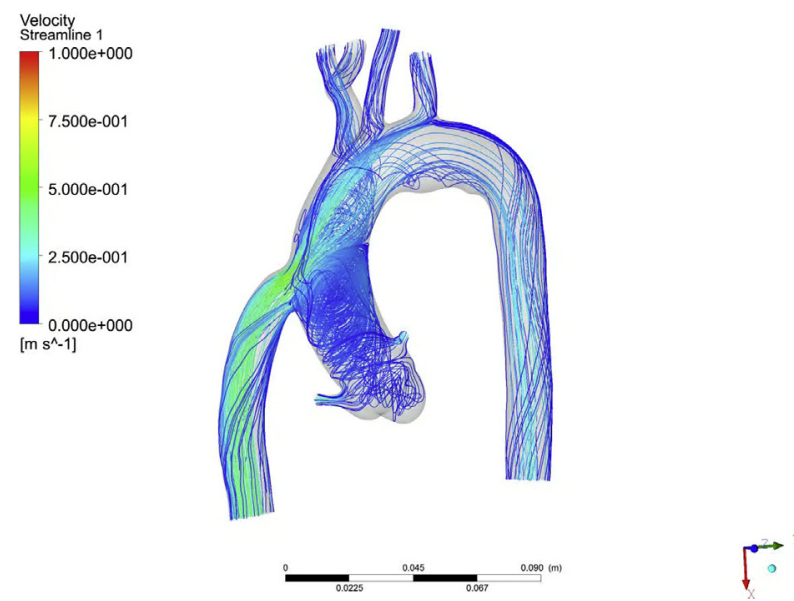

VIDEO 1. Streamline during 1 cardiac cycle from CFD simulation in a patient supported with a CF-LVAD showing retrograde spiral turbulence in the aortic root. Video available at: https://www.jtcvs.org/article/ S0022-5223(19)30284-3/fulltext. 

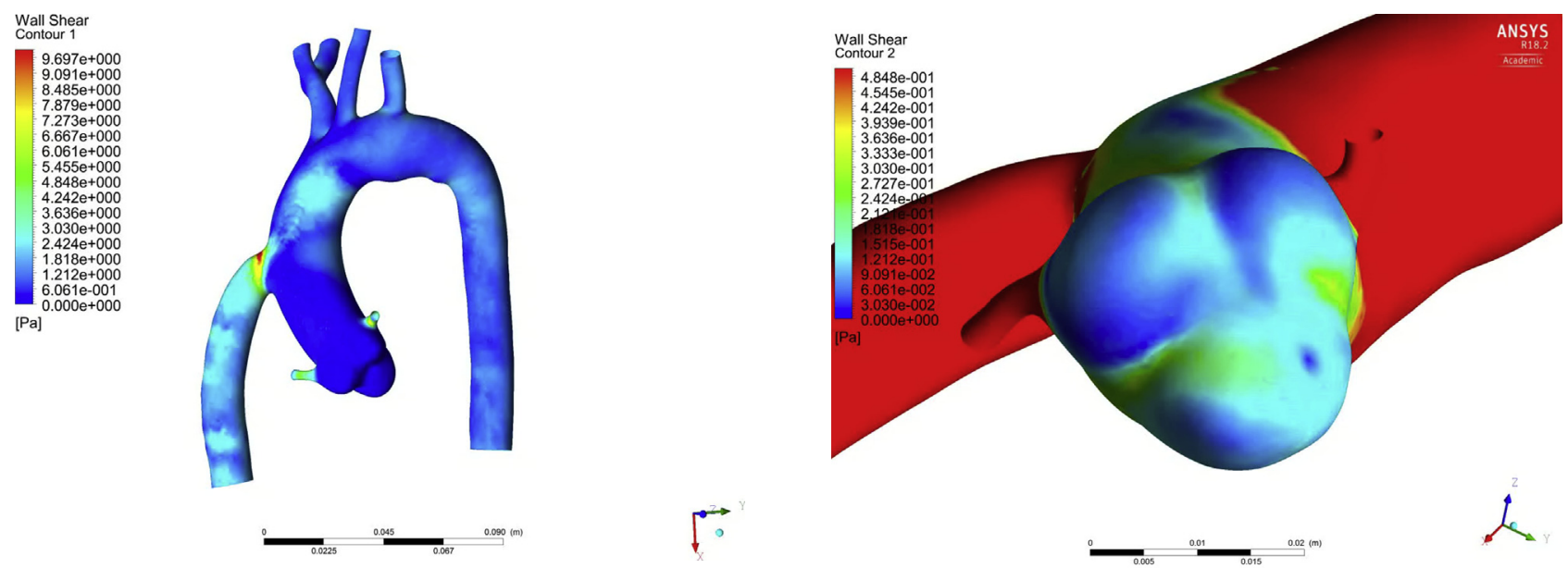

VIDEO 2. Shear stress during 1 cardiac cycle from CFD simulation in a patient supported with a CF-LVAD, showing low shear stress in the aortic root except in the coronary arteries. Video available at: https://www.jtcvs. org/article/S0022-5223(19)30284-3/fulltext.

Elucidation of the difference in blood flow pattern and shear stress between patients with and without de novo AI might allow preoperative CFD to predict AI occurrence after CF-LVAD implantation.

\section{References}

1. Bouabdallaoui N, El-Hamamsy I, Pham M, Giraldeau G, Parent MC, Carrier M, et al. Aortic regurgitation in patients with a left ventricular assist device: a contemporary review. J Heart Lung Transplant. 2018;37:1289-97.

2. Toda K, Fujita T, Domae K, Shimahara Y, Kobayashi J, Nakatani T. Late aortic insufficiency related to poor prognosis during left ventricular assist device support. Ann Thorac Surg. 2011;92:929-34.

VIDEO 3. Shear stress in the aortic valve during 1 cardiac cycle from CFD simulation in a patient supported with a CF-LVAD, showing that shear stress is similar in each aortic valve cusp and is relatively high in the commissures. Video available at: https://www.jtcvs.org/article/S00225223(19)30284-3/fulltext.

3. Hata H, Fujita T, Ishibashi-Ueda H, Nakatani T, Kobayashi J. Pathological analysis of the aortic valve after long-term left ventricular assist device support. Eur J Cardiothorac Surg. 2014;46:193-7.

4. Yoshida S, Fukushima S, Miyagawa S, Toda K, Sawa Y. Visualization of vortex flow and shear stress in the aortic root during left ventricular assist device support. J Thorac Cardiovasc Surg. 2017;154:877-8.e871.

5. Karmonik C, Partovi S, Loebe M, Schmack B, Weymann A, Lumsden $\mathrm{AB}$, et al. Computational fluid dynamics in patients with continuous-flow left ventricular assist device support show hemodynamic alterations in the ascending aorta. J Thorac Cardiovasc Surg. 2014;147: 1326-33.e1321. 